\title{
Tsafon
}

Revue d'études juives du Nord

$81 \mid 2021$

Des synagogues à travers les âges Lieux de prières, lieux d'études et autres fonctions

\section{Perego Simon, Pleurons-les. Les Juifs de Paris et la commémoration de la Shoah (1944-1967)}

\section{Rudy Rigaut}

\section{(2) OpenEdition}

\section{Journals}

Édition électronique

URL : https://journals.openedition.org/tsafon/4048

DOI : $10.4000 /$ tsafon.4048

ISSN : 2609-6420

Éditeur

Association Jean-Marie Delmaire

Édition imprimée

Date de publication : 1 juillet 2021

Pagination : 165-167

ISSN : $1149-6630$

Référence électronique

Rudy Rigaut, « Perego Simon, Pleurons-les. Les Juifs de Paris et la commémoration de la Shoah (1944-1967) », Tsafon [En ligne], 81 | 2021, mis en ligne le 01 juillet 2021, consulté le 15 septembre 2021. URL : http://journals.openedition.org/tsafon/4048 ; DOI : https://doi.org/10.4000/tsafon.4048

Ce document a été généré automatiquement le 15 septembre 2021

Tsafon. Revues d'études juives du Nord 


\title{
Perego Simon, Pleurons-les. Les Juifs de Paris et la commémoration de la Shoah (1944-1967)
}

\author{
Rudy Rigaut
}

\section{RÉFÉRENCE}

Perego Simon, Pleurons-les. Les Juifs de Paris et la commémoration de la Shoah (1944-1967),

Champ-Vallon, «Époques », oct. 2020, 392 p., $26 €$, numérique $18 €$.

1 Tiré de sa thèse de doctorat soutenue en 2016, l'ouvrage de Simon Perego traite des commémorations de la Shoah par les organisations juives parisiennes de 1944, date de la libération de la capitale, à la guerre des Six Jours en 1967. L'un des intérêts principaux de ce livre est de retranscrire de manière très fine le foisonnement de ces rassemblements, attestant ainsi de la reconstruction et du dynamisme de la vie juive à Paris dès la fin de la guerre. L'originalité méthodologique réside dans la variété des angles d'analyse et le déplacement permanent des échelles d'observation: entre commémorateurs et public; entre individus et groupements; entre public et privé ; entre espace et temps; entre local et général.

Dans l'introduction, l'auteur situe de manière habile son travail par rapport à l'historiographie de la première mémoire de la Shoah d'une part, et à celle des Juifs de France dans la deuxième moitié $\mathrm{du} \mathrm{XX}^{\mathrm{e}}$ siècle d'autre part. Il revient notamment sur les apports du livre Déportation et génocide d'Annette Wieviorka (1992) et sur la contestation $\mathrm{du}$ "mythe du silence" mettant en évidence la prise en compte précoce de la spécificité du sort subi. Outre l'analyse approfondie des rassemblements commémoratifs durant cette période, l'autre objectif est de proposer en parallèle une histoire des Juifs de Paris, un groupe présenté comme « restreint mais néanmoins dense et composite » (p. 21). 
Divisé en trois parties, le livre aborde de façon thématique l'ensemble des enjeux de la commémoration. Justifié par la constance de l'activité commémorative observée durant la période étudiée, le choix d'un plan thématique impose néanmoins, en raison de la méthodologie adoptée, la reprise systématique de certains éléments (calendrier, cadres spatiaux-temporels, ritualisation, analyse discursive etc.) dans les différentes parties. Nous allons tenter de les passer en revue.

4 Si tous les courants de ce que l'auteur appelle "l'archipel juif de Paris » se sont appropriés le geste commémoratif, contribuant ainsi à renforcer l'image d'un groupe unifié et homogène formant une "communauté commémorative ", la première partie révèle aussi comment la pratique de l'activité rituelle a été l'expression de la fragmentation de la judaïcité parisienne entre organisations religieuses, laïques, sionistes, bundistes, communistes; de ses conflictualités internes, exacerbées par le contexte de la Guerre froide; et de sa forte politisation. Simon Perego insiste avec raison sur les tensions politiques et idéologiques qui traversent cette collectivité. Quelques éléments comparatifs avec d'autres groupes juifs du territoire national auraient permis de mieux faire ressortir cette spécificité locale. Par ailleurs, l'étude de l'élaboration du calendrier commémoratif est l'occasion de rappeler la précocité des rassemblements honorant la mémoire des victimes du génocide. Ainsi, le premier de ces rassemblements a été organisé par l'association cultuelle israélite de Paris à partir de 1946 à la grande synagogue de la rue de la Victoire. Impliquant diverses organisations juives locales (communistes, sionistes, religieux, bundistes, etc.) d'autres cérémonies se déroulent dont certaines sont dédiées à des événements particuliers, tels que le soulèvement du ghetto de Varsovie, afin d'entretenir aussi la mémoire de la résistance des Juifs. Le choix des lieux devenus des sites commémoratifs comme la cité de la Muette à Drancy, le Vel' d'Hiv' ou le Mémorial du martyr juif inconnu permet à l'auteur d'appréhender de manière pertinente la question de la spatialité de cette pratique.

5 Adoptant une approche culturelle et religieuse de la mémoire, la deuxième partie porte sur les enjeux mémoriels revêtus par ces commémorations. Dans un premier temps, les cadres spatio-temporels et la ritualisation de l'hommage aux victimes ont été abordés. Simon Perego montre comment, dès l'immédiate après-guerre, le calendrier religieux a été mobilisé par les autorités consistoriales pour adopter une date spécifiquement dédiée à la commémoration de toutes les victimes juives. Fixée le dimanche précédant le nouvel an juif, « la cérémonie eut lieu pour la première fois le 22 septembre 1946 et fut reconduite chaque année jusqu'à aujourd'hui » (p. 140). La dimension spatiale de l'hommage révèle l'importance de ces lieux qui représentent, aux yeux des survivants et des endeuillés, « des tombes de substitution » (p. 147). Quant à la ritualisation, elle se caractérise par l'intégration d'éléments religieux (récitation de prières, usage des bougies) mais aussi par la place considérable accordée à la conservation des noms. Autre point important de cette deuxième partie, la fabrique mémorielle est appréhendée à travers l'étude des discours, de la terminologie employée, des événements commémorés et de leur interprétation. Si le sort spécifique des Juifs est affirmé très tôt, l'auteur observe aussi une « tentation de l'universalisation » (p. 177) et une appropriation de la vision globalisante des crimes nazis par certains acteurs de la scène commémorative juive à Paris. Enfin, l'analyse de l'enjeu de la transmission mémorielle met l'accent sur la volonté des organisateurs d'associer la jeunesse à ces manifestations. En ayant recours à des carnets personnels et/ou à des entretiens, le 
sujet aurait pu être aussi abordé du point de vue des enfants afin de questionner leurs représentations, leur engagement et leur réceptivité.

6 Les commémorations représentent aussi des moments particuliers où les appartenances identitaires peuvent s'affirmer publiquement. Dans cette dernière partie, trois pôles d'identification ont été retenus. Le premier est celui de la religion. L'auteur souligne la volonté des dirigeants consistoriaux d'ancrer les rassemblements dans la vie cultuelle. La commémoration devient même pour les plus éloignés de la religion le dernier lien avec une forme de sacralité. S'appuyant sur le cas du Mémorial du martyr juif inconnu, des pages très intéressantes sont consacrées aux rapports étroits et parfois conflictuels qu'ont pu entretenir religion et commémoration. L'appartenance française représente le deuxième pôle étudié. La participation des pouvoirs publics, des représentants des grands partis politiques et des "non-Juifs » revêt alors une dimension symbolique forte. Un monument commémoratif à l'intérieur de la grande synagogue de la rue de la Victoire a été inauguré le 27 février 1949 par le président de la République Vincent Auriol. Fait très intéressant, lors des discours, tous les sujets sont déjà évoqués, celui plus glorieux des "Justes" visant à valoriser l'engagement de Français dans le sauvetage de Juifs mais aussi celui encore très sensible de la complicité de la France et des responsabilités du régime de Vichy. En revanche, la réflexion sur l'utilisation de la catégorie des "non-Juifs» et la confusion possible entre religion et nationalité chez les organisateurs ou dans les articles de presse aurait pu être davantage approfondie. Enfin, la référence à Israël constitue le troisième et dernier pôle d'identification abordé. Les prises de parole lors des commémorations organisées dans les années d'après-guerre par les sionistes, les bundistes ou les communistes reflètent les divergences d'opinion qui existent alors au sein de la judaïcité parisienne à propos de l'État d'Israël. Des liens ont pu être établis par les orateurs entre la Shoah et les dangers encourus par le pays. Enfin, les dernières pages reviennent notamment sur l'existence d'une concurrence commémorative entre le projet de mémorial à Jérusalem et celui d'Isaac Schneersohn à Paris.

7 Il est toujours possible de discuter de la densité de l'ouvrage qui s'adresse prioritairement à des lecteurs avertis, de questionner le choix des limites chronologiques ou celui d'un cadre spatial trop parisiano-centré. Cependant, l'ouvrage de Simon Perego invite aussi à une véritable réflexion sur ces processus dans d'autres régions du territoire national à l'image de ce que nous avons modestement tenté de faire dans le dernier chapitre de notre thèse de doctorat consacrée aux logiques d'identification et d'appartenance des Juifs dans la zone littorale du Nord et du Pas-deCalais. Surtout, les très nombreux apports historiographiques, la combinaison des points de vue mobilisés, l'utilisation d'une grande diversité de fonds d'archives, la collecte massive de données et la maitrise solide de la bibliographie reflètent la qualité scientifique de ce livre qui s'impose désormais comme une étude de référence sur la mémoire de la Shoah en France. 